\title{
Espectro De CORES DO PONTO DE Vista MATEMÁtico.
}

\author{
Fernando Antonio de Araujo Carneiro, ${ }^{\dagger}$ Leandro Mendes Barrigio.
}

\begin{abstract}
Resumo
Apresentamos a história da representação matemática do espectro de luz visível. com modelos algébricos e geométricos do espaço de cores, e alguns exemplos de problemas de mistura de cores. Apresentamos a primeira representação, na Grécia Antiga, os vários modelos propostos por Newton, Mayer, Lambert, Maxwell, Munsell e Grassmann, e os sistemas RGB e CMYK.
\end{abstract}

\begin{abstract}
We summarize the history of the mathematical representation of the visible light spectrum. We present algebrical and geometrical models for the space of colours and problems about colour mixing. We introduce the first representation in Ancient Greece, the color spaces of Newton, Mayer, Lambert, Maxwell and Grassmann, and finally the RGB and CMYK systems.
\end{abstract}

*Palavras-chave: Espectro de cores, Geometria, Matemática Aplicada.

†Professor Adjunto do Departamento de Geometria e Representação Gráfica, IME-UERJ, carneiro@ime.uerj.br

DOI: https://doi.org/10.12957/cadmat.2019.37888 


\section{Introdução}

Por volta do século XII, no ambiente escolástico, iniciou-se uma discussão quanto ao problema da latitude das formas, dentro do qual se encaixa a quantificação das cores. O problema consiste no estudo de variações de intensidades de qualidades, como cores, temperatura, movimento, entre outras. Para explicar melhor, pensemos na longitude como sendo uma extensão: um período de tempo - a duração de um movimento - ou uma magnitude corporal - como a superfície de um corpo. A latitude corresponde à intensidade de uma qualidade: a temperatura de um corpo, a intensidade de luz de uma fonte, ou a velocidade de um corpo em movimento. Edith Sylla [6] afirma que uma das fontes da ideia de se quantificar qualidades na Idade Média veio dos estudos de óptica, mais especificamente dos estudos da variação da intensidade da luz de acordo com a sobreposição de mais de uma fonte iluminante, o que teria influenciado Oresme (1323-1382) a criar um modelo geométrico para as variações de intensidade ver Figura 1.

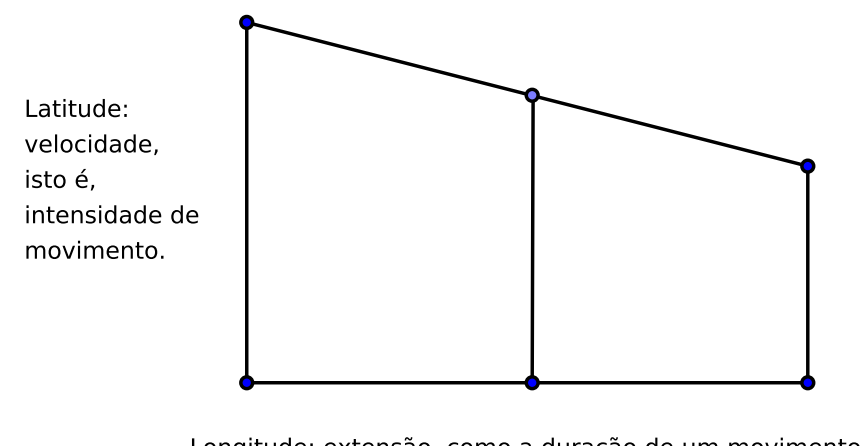

Figura 1: Modelo geométrico de Oresme.

O momento histórico medieval talvez tenha sido o ponto de partida da posterior quantificação cada vez maior do que antes era visto como qualidades, em contraposição às quantidades, categoria distinta da primeira no conjunto de categorias aristotélicas. Segundo os antigos, o estudo de uma não se adequava ao da outra. Apesar disso, é bem antigo o estudo não somente da luz como do espectro de cores.

A partir da quantificação cada vez maior, o estudo do espectro de cores adquiriu cada vez mais um aspecto geométrico e numérico.

Na segunda seção, vamos falar do desenvolvimento histórico do espaço de cores e suas etapas. A primeira etapa, que se inicia nos gregos e segue até os experimentos de Isaac Newton, se caracteriza pela tentativa ainda simples de ordenar as cores mais básicas. São ordenações lineares, baseadas em 
outras ordenações ou disposições em sequência de elementos do mesmo tipo, como as notas musicais, cuja ordem é justificadamente linear. A segunda etapa, iniciada por Newton e que vai até Grassmann, surge dos experimentos de decomposição da luz, que indicam que as cores têm um caráter combinatório, e é caracterizada pela tentativa de não só determinar as cores básicas, de cujas combinações surgem as outras cores, como determinar o espaço de cores, uma espécie de espaço de possibilidades das cores. Nessa etapa surgem o círculo de Newton, o triângulo de Maxwell e o prisma de Lambert. A terceira etapa é dada pelas contribuições de Munsell e Hermann Grassmann, o primeiro ao distinguir os elementos a ser quantificados, matiz, saturacão e brilho, e o segundo, não somente ao metrizar o espaço de cores, mas ao determinar a quais axiomas a combinatória das cores obedeceria, o que permitiu identificar cores e coordenadas num espaço.

$\mathrm{Na}$ terceira seção falamos do espaço de cores aditivo e subtrativo - o primeiro se relaciona com a decomposição da luz, o segundo com misturas de pigmentos. Nesta seção apresentamos alguns exemplos de problemas envolvendo os dois espaços.

\section{Desenvolvimento histórico do espaço de cores}

Da Grécia antiga temos a escala de cores de Aristóteles (384 a.C.-382 a.C.), que as representa como um grupo linear de sete cores - ver Figura 2 -, muito provavelmente por uma influência pitagórica que as relacionava aos planetas e às notas musicais, relação por trás da qual havia o princípio da harmonia universal. O sistema de cores aristotélico foi utilizado até surgir o sistema de cores de Isaac Newton (1643-1727).

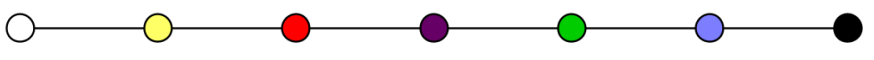

Figura 2: Escala de cores de Aristóteles.

Mas, antes do surgimento do sistema de cores newtoniano, por volta de 1613, o matemático e físico François de Aguillon (1567-1617) distinguiu três tipos de cores em sua obra de óptica geométrica [1], [4], [5]. Seu sistema era linear como o de Aristóteles mas ele escolheu o vermelho, o amarelo e o azul como as principais cores. Esse é o primeiro caso em que são utilizadas três cores básicas, o que se tornaria uma propriedade constante de muitos dos sistemas posteriores.

Ao fazer seus experimentos da decomposição da luz que passa por um prisma - ver Figura 3 -, Isaac Newton percebeu que as cores se dispõem em um círculo que contém as visíveis saturadas, o que acrescenta ao esquema linear de Aristóteles mais uma dimensão. O matemático e astrônomo Tobias Mayer (1723-1762) procurou identificar o número de cores que o olho humano é capaz de perceber, a 


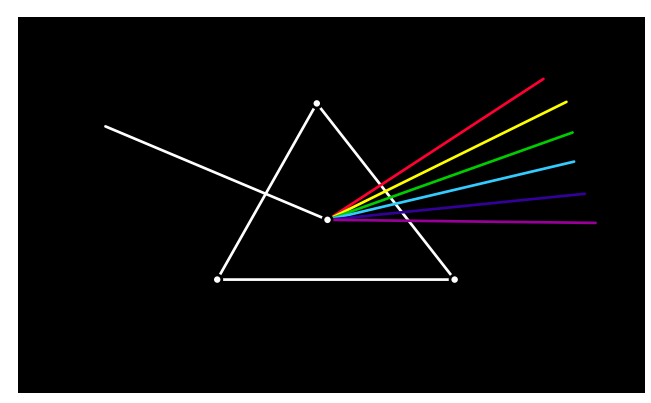

Figura 3: Decomposição da luz branca por um prisma.

partir do plano de cor de Newton, se utilizando da regra de que uma cor está no centro de gravidade de um segmento entre duas cores originais; mas seu modelo não era circular e sim triangular, com o vermelho, amarelo e azul como vértices do triângulo, e é o primeiro exemplo de espaço métrico de cores, isto é, um espaço onde a posição da cor tem relação com a sua proporção como mistura de cores primárias [1], [4], [5] - ver Figura 4.

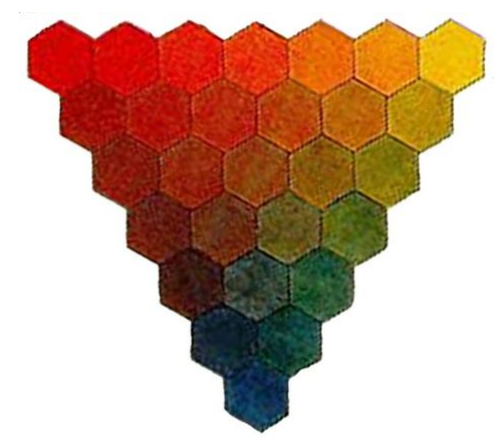

Figura 4: Triângulo de Mayer.

Fonte: https://www.pinterest.com/pin/321866704614203621/

A seguir, o matemático Johann Heinrich Lambert (1728-1777) acrescentou profundidade ao modelo de Mayer, que deixava de fora a luminosidade, gerada pelos graus de brancura; Lambert sugeriu o modelo em forma de uma pirâmide formada por vários triângulos de Mayer emelhantes, tendo a cor preta como centro de sua base, já que o vermelho, o amarelo e o azul, misturados em certas proporções, geram o preto, e a cor branca no vértice oposto à base formada pelo triângulo de Mayer - ver Figura 5. Daí que Lambert seja considerado o descobridor do sistema de mistura de cores subtrativo [1].

Vale mencionar a contribuição do médico Thomas Young, o primeiro a sugerir que a retina dividia o espectro de cores em três partes básicas, ou seja, há não somente três tipos de luz mas três tipos de sensações cromáticas elementares: o que vem a ser hoje em dia conhecido como a Teoria tricromática da visão, cujo estudo quantitativo foi realizado por James Clark Maxwell (1831-1879) [4]. 


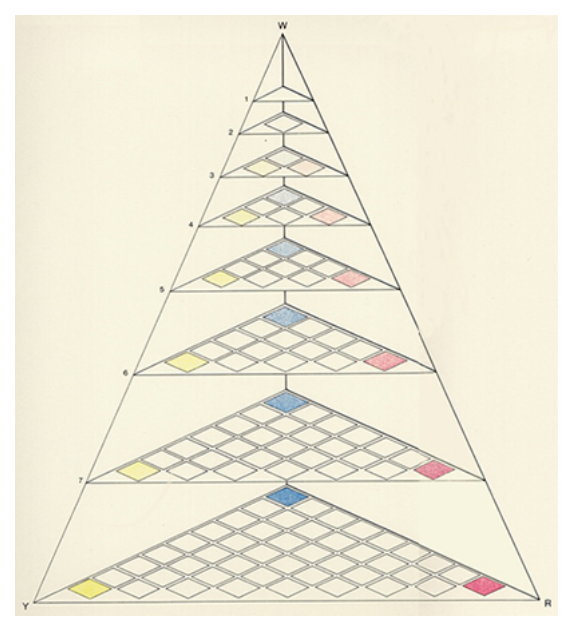

Figura 5: Modelo de Lambert.

Fonte: https://www.colorsystem.com/

Albert Henry Munsell (1858-1918) identificou os parâmetros matiz, valor e croma, a partir dos quais criou sua Árvore de Cor, de cujos métodos físicos hoje em dia todos os pesquisadores desse fenômeno se utilizam. Para esclarecer, o valor é o brilho, a luminosidade da cor, ou seja, a intensidade na escala que vai do escuro ao claro; o croma é a saturação ou intensidade, se referindo não à intensidade de luminosidade mas à intensidade de vivacidade da cor; o matiz é o tom da cor, a posição da cor na escala de cores básicas de Newton - a Figura 6 abaixo ilustra os parâmetros das cores segundo eixos distintos.

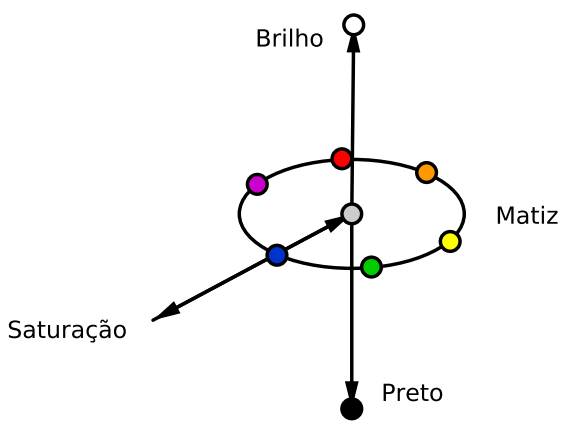

Figura 6: Árvore de cores de Munsell.

Hermann Grassmann (1809-1877), ao revisar o círculo de Newton, e ao reabilitar seu método do centro de gravidade, propôs que as sensações de cores se relacionam com misturas de luz de acordo com alguns princípios. Uma das diferentes formulações das leis de Grassmann é a seguinte: 
i. O brilho, a saturação e matiz de duas ou mais luzes preveem o brilho, saturação e matiz de sua mistura.

ii. Quaisquer duas luzes que parecem ser da mesma cor misturam cores idênticas com qualquer terceira luz, mesmo que os perfis espectrais de das luzes contenham comprimentos de onda em diferentes proporções.

iii. Se duas misturas de luzes têm uma cor idêntica e, em seguida, lhes adicionamos uma terceira luz, na mesma intensidade, as misturas resultantes serão idênticas.

iv. A cromaticidade (tonalidade e saturação) de qualquer luz pode ser compensada por um ou dois comprimentos de onda monocromática misturada com alguma quantidade de luz branca (acromática).

Grassmann também propôs uma versão quantitativa, pois considerava que as cores seriam aditivas no matiz, brilho e croma, elementos que podem ser matematicamente determinados. Essa mistura pode ser representada por somas geométricas de pesos a e b dados a pontos A e B no espaço de cores.

Sejam as cores X, Y, Z e W, as seguintes relações são verdadeiras:

- Simetria: Se $\mathrm{X}=\mathrm{Y}$ então $\mathrm{Y}=\mathrm{X}$;

- Transitividade: Se $\mathrm{X}=\mathrm{Y}$ e $\mathrm{Y}=\mathrm{Z}$ então $\mathrm{X}=\mathrm{Z}$;

- Proporcionalidade: Se $\mathrm{X}=\mathrm{Y}$ então $\mathrm{X} . \mathrm{a}=\mathrm{b} . \mathrm{Y}$ para todos os números reais não nulos a e b, a=b;

- Aditiva: Se $\mathrm{X}=\mathrm{Y}$ e $\mathrm{X}+\mathrm{Z}=\mathrm{Y}+\mathrm{W}$ então $\mathrm{Z}=\mathrm{W}$.

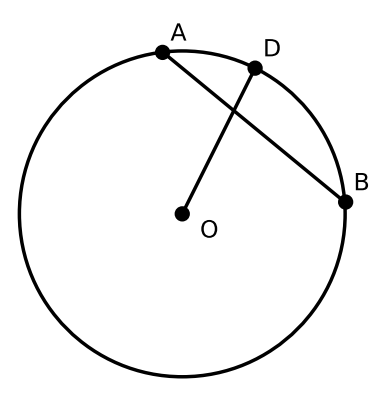

Figura 7: Exemplo de aplicação dos princípios de Grassmann.

Qualquer ponto do segmento $A B$ da figura acima representa uma mistura das cores associadas aos pontos $A$ e $B$ do locus do espectro. Desse ponto traçamos o segmento que passa pelo ponto branco $O$ e que corta o locus do espectro em $D$. Essa cor também pode ser vista como a mistura de uma cor do locus do espectro com o branco. Daí concluímos que a cor do ponto $D$ representa a matiz da mistura. A saturação será dada pela distância do ponto ao ponto $O$. 
A partir do século XX, dados os vários estudos sobre o processo visual e o pedido de padronização feito pela Comissão Internacional de Iluminação (CIE), o físico Deane Brewster Judd (1900-1972) estabeleceu um observador padrão para o sistema tricromático de cores segundo valores triestimulares. O diagrama de cromaticidade, cuja base é o triângulo de Maxwell, resulta da medição da absorção dos cones dos olhos diretamente do espectro de cores.

\section{Os espaços de cores aditivo e subtrativo.}

O espectro de cores é linear e a posição no espectro varia de acordo com o comprimento de onda. Já o espaco de cores é representado de duas maneiras: valores nulos do espectro são atribuídos ao tom preto, e o espectro é projetado na superfície plana de um triângulo equilátero, cujos vértices representam os valores máximos de três cores primárias. O locus do espectro tridimensional é projetado em duas dimensões e o espaço obtido é um triângulo retângulo. Um segmento de reta da origem até o ponto branco forma uma escala de cinza (ausência de cor).

O triângulo de Maxwell representa as diferenças de matiz e grau de pureza e não representa a luminosidade. O interior do triângulo é chamado de diagrama de cromaticidade, cujos vértices são cores de máxima pureza. Qualquer ponto no interior do triângulo representa uma cor que resulta da mistura de três cores básicas. O ponto branco é aquele tal que a contribuição das cores primárias, vermelho, verde e azul é a mesma, ou seja, cada cor primária contribui em um terço. A pureza de uma cor é dada pelo comprimento do segmento entre o ponto branco e o ponto da cor. As misturas fora do triângulo são cores impossíveis de se realizar.

Seguindo a determinação de Newton de que a cor é uma propriedade perceptual e não um atributo físico, hoje a cromaticidade é definida como misturas de estímulos proporcionais do espaço de cores LMS 1 de acordo com a sensibilidade dos três tipos de cones: o primeiro responde à luz de comprimentos longos, associada à cor vermelha; o segundo a de comprimentos médios, de cor verde; o terceiro a de comprimentos curtos, azulada.

\footnotetext{
${ }^{1}$ Espaço baseado na percepção das cores pelo olho através dos três tipos de cones [4].
} 
Por exemplo, qual é a cor resultante ao misturarmos quantidades iguais de 490 nanômetros e 600 nanômetros?

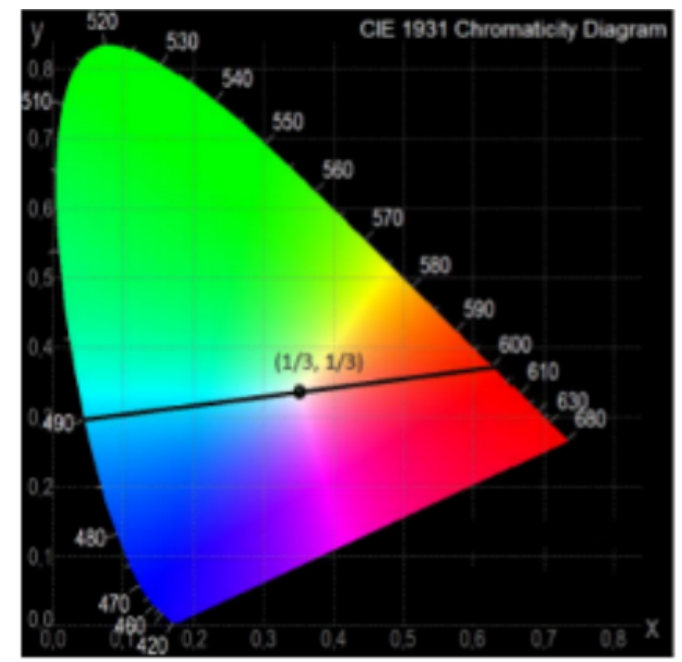

Figura 8: Diagrama de cromaticidade.

Fonte: o segundo autor, 2016. Software CIE Chromaticity Diagrams.

Como 490 e 600 nanômetros são lados opostos do locus do espectro e estão em quantidades iguais, a mistura produz o branco - ver Figura 8 acima.

Já o efeito visual da cor ao misturarmos substâncias materiais, como no caso de tintas, não é o aumento de luz refletida mas sim o seu comportamento na absorção da luz, cujos comprimentos de onda se reduzem ao ser absorvidos, o que reduz o brilho nas coisas materiais. Ao misturarmos as três cores primárias produzimos cor escura, o oposto do branco. Por isso a mistura reduz a matiz, aumenta o cinzento da mistura. Além disso, e também por essa razão, a mistura reflete somente os comprimentos de onda que ambas as cores misturadas refletem. Elas formam um espaço de cores subtrativo.

Cada cor primária subtrai do branco luz dos comprimentos de onda que representam uma cor primária. As fórmulas subtrativas envolvendo o branco $(\mathrm{W})$, o preto $(\mathrm{K})$ e as cores primárias vermelho $(\mathrm{R})$, verde (G) e azul (B) são:

- $\mathrm{W}-\mathrm{B}=(\mathrm{R}+\mathrm{G}+\mathrm{B})-\mathrm{B}=\mathrm{R}+\mathrm{G}=\mathrm{Y}$ (amarelo);

- $\mathrm{W}-\mathrm{G}=(\mathrm{R}+\mathrm{G}+\mathrm{B})-\mathrm{G}=\mathrm{R}+\mathrm{B}=\mathrm{M}$ (magenta)

- $\mathrm{W}-\mathrm{R}=(\mathrm{R}+\mathrm{G}+\mathrm{B})-\mathrm{R}=\mathrm{G}+\mathrm{B}=\mathrm{C}($ ciano $)$;

- $\mathrm{W}-(\mathrm{R}+\mathrm{G}+\mathrm{B})=\mathrm{K}$ (preto).

Um exemplo de problema que envolve o espaço subtrativo: uma luz magenta brilha sobre uma folha de papel contendo pigmento amarelo. Qual seria a aparência no papel? A luz magenta consiste de luz 

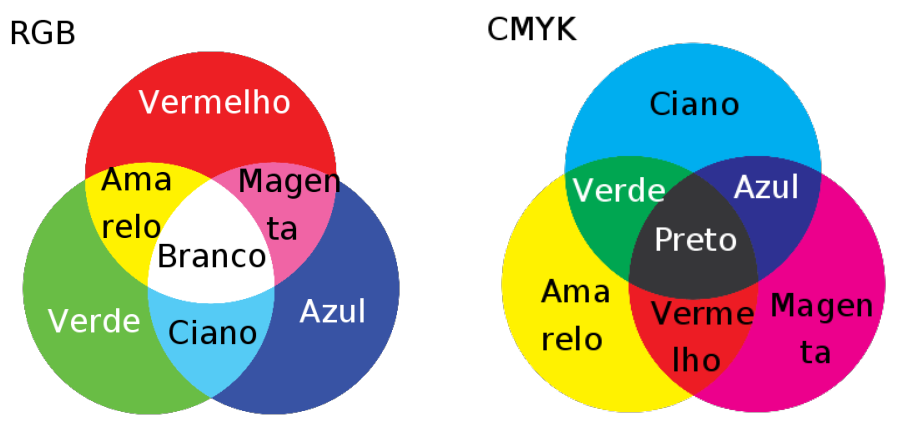

Figura 9: Sistemas de cores, o aditivo RGB à esquerda, o subtrativo CMYK à direita.

vermelha e luz azul. O amarelo absorve a luz azul, seu complementar. Assim, azul é subtraído da luz que incide sobre o papel, refletindo a luz vermelha.

$\mathrm{M}+\mathrm{Y}=(\mathrm{R}+\mathrm{B})+(\mathrm{R}+\mathrm{G})=((\mathrm{R}+\mathrm{G}+\mathrm{B})-\mathrm{G})+((\mathrm{R}+\mathrm{G}+\mathrm{B})-\mathrm{B})=\mathrm{W} \quad(\mathrm{G}+\mathrm{B})=\mathrm{R}$.

\section{Conclusão}

A história da representação do espectro de cores nos mostra como, ao passarmos a representar quantitativamente as intensidades das cores (que não são únicas já que são independentes tanto a intensidade de luminosidade quanto a intensidade de saturação) avançamos na matematização das cores quando tentarmos representá-las num espaço cuja estrutura reflete as relações entre elas e suas intensidades.

\section{Referências}

[1] BARRIGIO, L. M., Representação matemática do espectro de luz visível, , Dissertações de Profmat Ime-Uerj, SBM, 2016.

[2] CLAGETT, MARSHALL, Nicole Oresme and the medieval geometry of qualities and motions. A treatise on the uniformity and difformity of intensities known as tractatus de configurationibus qualitatum et motuum, University of Wisconsin Press: Madison, Milwaukee, 1968.

[3] DUHEM, PIERRE, Le système du Monde, histoire des doctrines cosmologiques de Platon à Copernic, tomes VII et VIII. Paris, Hermann, 1956-1958

[4] KUEHNI, R. G. Color Space and Its Divisions: Color Order from Antiquity to the Present. New Jersey: John Wiley \& Sons, Ltd, 2003.

[5] KUEHNI, R. G; SCHWARTZ, A. Color Ordered: A Survey of Color Order Systems from Antiquity to the Present. New York: Oxford University Press, 2008. 
[6] SYLLA, EDITH, Medieval Quantifications of Qualities: The "Merton School", Archive for History of Exact Sciences Vol. 8, No. 1/2 (30.XII.1971), pp. 9-39 (31 pages) 